exothermic peak is unaffected. Activation energy for the main dehydration process calculated from the differential thermal curves is about $40 \mathrm{kcal}$. for the rehydrated kaolin, compared with $55 \mathrm{kcal}$. for the original material. The slope ratio of the main endothermic peak is 4-1 for the rehydrated material,'a figure which has been thought characteristic of halloysite ${ }^{1}$. The slope ratio for the untreated kaolin is 1.25-1:

Preliminary tests on other materials indicate that the hardening effect occurs in relatively well-crystallized kaolins.

Possible industrial applications of the phenomenon are being investigated.

Division of Building Research,

Commonwealth Scientific and

Industrial Research Organization, Highett, S.21,

Victoria, Australia.

Sept. 18.

${ }^{1}$ Bramao, L., Cady, J. G., Hendricks,'S. B., and Swerdlow, M., Soil. Sci., 78,273 (1952).

\section{Centrifugation Potentials in Sols of Silver lodide}

IF a sol is centrifuged, an electrostatic potential difference is set up between two points in the sol at different distances from the axis of rotation (centrifugation potential) ${ }^{\mathbf{1}}$. These potentials are connected with the $\zeta$-potential of the colloidal particles by the formula :

$$
V_{\text {C.P. }}=\frac{m^{\prime} n}{\sigma} \frac{D \zeta}{6 \pi \eta} \frac{\omega^{2}}{2}\left(R_{2}^{2}-R_{1}^{2}\right) .
$$

Here $m^{\prime} n$ is the apparent mass of dispersed substance per cm. ${ }^{3}$, $\sigma$ the electrical conductivity, $\eta$ the viscosity, $D$ the dielectric constant of the sol, $\omega$ the angular speed of rotation, and $R_{2}, R_{1}$, the distances from the axis of the two points between which the centrifugation potential is measured. Conversely, from the value of $V_{\text {C.P. }}, \zeta$ can be calculated; therefore the measurement of centrifugation potentials is of considerable interest.

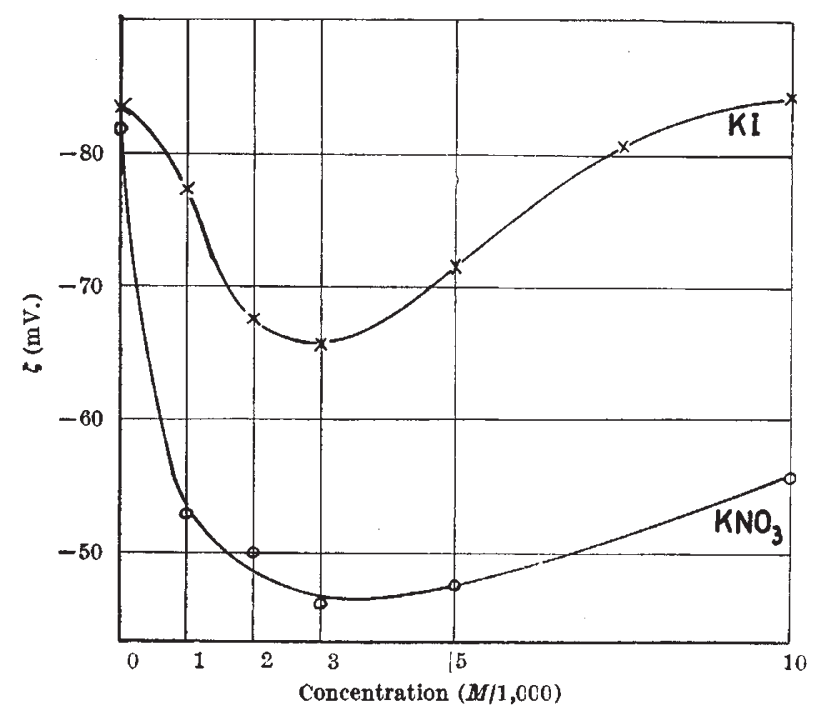
Values of $\zeta$ in sols of silver iodide after the addition of potassium nitrate
and of potassium iodide
Extensive measurements of these potentials in sols of arsenic trisulphide have been made by G. Jacobs ${ }^{2}$. One of his results, and certainly the most unexpected, was the shape of the $\zeta-c$ curves for the addition of indifferent electrolyte (potassium nitrate). Between 0 and 3 millimol. $/ 1 ., \zeta$ decreases from -100 to -73 $\mathrm{mV}$. and then rises slowly again.

We decided to repeat these measurements, in order to see whether Jacobs's result was a general one, for sols of silver iodide. To obtain the necessary quantities a continuous electrodialyser was constructed ${ }^{3}$. We prepared a concentrated sol, containing $50 \mathrm{gm} . / 1$. of silver iodide; the coarsest particles were eliminated by pre-centrifugation, resulting in a sol of about $20 \mathrm{gm}$. $/$. with which the measurements were made.

The results are shown in the accompanying graph; the reproducibility of the points was about 3 per cent. There is again a strong decrease of $\zeta$, followed by a slow increase, for the addition of indifferent electro lyte; for the addition of potential-determining electrolyte, there is also a decrease of $\zeta$, followed by a much more marked increase. This last feature is in agreement with expectation, and gives us confidence in the experimental procedure; the first result is quite unexpected, and, so far as we are aware, cannot be explained by the current theories of the double layer. In fact, these theories always lead to a monotonic change of $\zeta$ with concentration.

We are proposing to make simultaneous measurements of $\zeta$ and $\varepsilon$ (the total potential) in order to obtain more insight into this problem.

$$
\begin{aligned}
& \text { A. J. Rutgers } \\
& \text { P. Nagels }
\end{aligned}
$$

Laboratory of Physical Chemistry, University, Ghent. Sept. 10.

${ }^{1}$ Rutgers, A. J., Nature, 157, 74 (1946).

Jacobs, G., Trans. Farad. Soc., 48, 355 (1952).

s Rutgers, A. J., and Swyngedouw, R., Nature, 168, 727 (1951).

\section{Curves of Succinoxidase Inhibition by Surface-active Agents}

INHIBITION of the succinoxidase system by a variety of surface-active agents has been noted by Hockenhull ${ }^{1}$. The inhibition of this enzyme system by certain water-soluble androgens has recently been demonstrated ${ }^{2}$. The effect of these androgens suggested a surface phenomenon ${ }^{3}$.

Two detergents were therefore studied in this laboratory to compare curves of inhibition with those found for the androgens. Sodium deoxycholate was chosen because it is both an ionic detergent and a soluble steroid salt. Polyoxyethylene sorbitan monolaurate ('Tween 20') was the neutral detergent used. The manometric technique employed was that described in Umbreit's text-book ${ }^{4}$. The accompanying curves (Figs. 1 and 2) show the influence of detergent concentration on the succinoxidase system of rat liver.

The sigmoid shape of these curves is similar to that of curves obtained when sodium androsterone sulphate is the inhibiting agent. If we consider per cent inhibition analogous to uptake of inhibitor by the enzyme system, we may compare the curves obtained here with adsorption curves in other systems. For example, Coolidge $e^{5}$ found this type of curve for the adsorption of water vapour by charcoal under conditions of increasing vapour pressure. Evidence derived from the work with androgens indicates that 